\title{
Report of a new grape powdery mildew morphotype with branched conidiophores
}

\section{Riaz $\mathrm{S}^{1}$, Braun $\mathrm{U}^{2}$, Lejkina $\mathrm{I}^{1}$, Gubler $\mathrm{WD}^{3}$ and Walker $M A^{* 1}$}

\author{
${ }^{1}$ Department of Viticulture and Enology, University of California, Davis, CA 95616, \\ ${ }^{2}$ Martin-Luther-Universität, Institut für Biologie, Bereich Geobotanik und Botanischer Garten, Herbarium, Neuwerk \\ 21, D-06099 Halle/S. Germany \\ ${ }^{3}$ Department of Plant Pathology, University of California, Davis, CA 95616,
}

Riaz S, Braun U, Lejkina I, Gubler WD, Walker MA 2013 - Report of a new grape powdery mildew morphotype with branched conidiophores. Plant Pathology \& Quarantine 3(1), 19-27, doi $10.5943 / \mathrm{ppq} / 3 / 1 / 4$

We report a new morphotype of Erysiphe necator, the fungal pathogen cause of grapevine powdery mildew. Compared to normal isolates, the new morphotype develops the first conidium on the tip of conidiophore by day five after inoculation and stays in arrested growth phase until day 9 or 10 . On day 10 or 11, a branch appears at the base of first conidium that independently starts making conidia. Both main and side branches of conidiophores develop chains that are short with 2-4 conidia, their conidia take a longer time to mature, and they have stronger adhesion to sister conidia on the chain. The branching process starts from the centre of the colony and moves to the edges. Mature colonies have a heterogeneous appearance with non-branched conidiophores at the edges of the colony by day 18 . The number of conidiophores produced by the new morphotype as compared to a normal unbranched isolate was not significantly different.

Key words - Conidium - Erysiphe necator - hyphae - mycelium - Vitis vinifera

\section{Article Information}

Received 28 April 2013

Accepted 9 May 2013

Published online 8 June 2013

*Corresponding author: M. Andrew Walker - awalker@ucdavis.edu

\section{Introduction}

Grape powdery mildew caused by Erysiphe necator Schwein. [syn. Uncinula necator (Schwein.) Burrill] is one of the most serious pathogens facing grape growers. It colonizes and infects all herbaceous tissues on susceptible grapevines. Young colonies are whitish in appearance, roughly circular, and occur singly or in groups (Gadoury et al. 2012). Normally, perpendicular translucent multiseptate conidiophores arise from superficial hyphae in growing colonies. Conidiophores may also occasionally be formed via microcyclic conidiogenesis, where germinating conidia produce conidiophores directly (Kiss et al. 2008, Cook \& Braun 2009). The conidiophores of E. necator are of the Pseudoidium type, i.e. the conidia mature singly, although the conidia may adhere in "false chains" when the humidity is high and the air still (Braun \& Cook 2012). Conidia are colorless, uninucleate, and glassy translucent. Ellipsoid-ovoid to doliiform single conidia are dispersed by air movement and other mechanical means.

Conidia germinate easily on superficial substrates (e.g., glass, water, agar). On the surface of a susceptible host plant, they generate a primary, true germ tube that forms a terminal lobate primary appressorium. The 

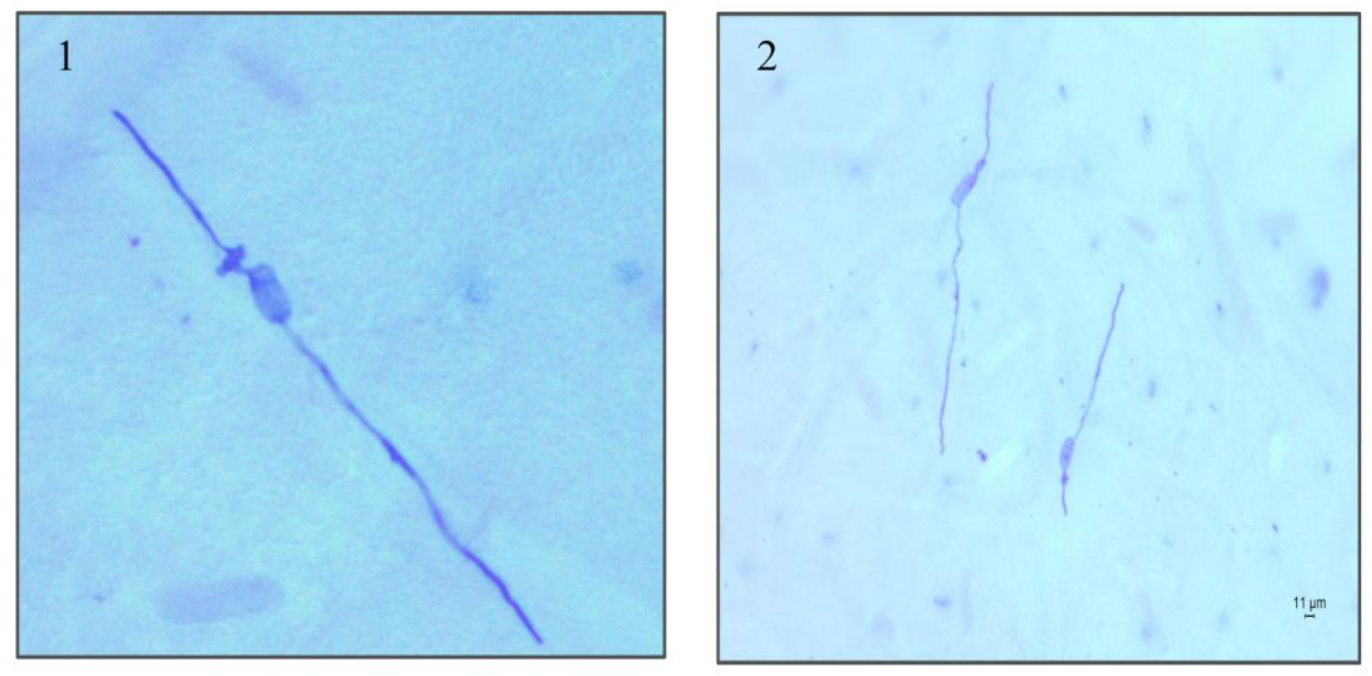

Fig. 1 - Conidium germination 24 hpi. 1 Isolate with branched conidiophores. 2 C-isolate.

point of origin of the germ tube is thought to be predetermined (Takikawa et al. 2011) and is not impacted by where the conidia first touch the host surface. Primary hyphae grow from the other side of the conidium as well as from the primary appressorium to initiate colony formation. Ascomata or chasmothecia (fruiting bodies) develop when hyphae of compatible mating types come in contact (Braun \& Cook 2012). Features of the mature ascomata have been used to classify mildew genera.

This study reports on a new morphotype of E. necator that forms branched conidiophores. The branching initiates at the base of first conidium on the conidiophore; further growth results in the development of chains of conidia on the initial and secondary branches. This phenomenon has not been reported for grape powdery mildew before. We observed this behavior in isolates collected from three different sites. One isolate of the new morphotype was purified and used to compare growth patterns with a normally developing powdery mildew isolate.

\section{Methods}

\section{Collection and maintenance of powdery mildew isolates}

Isolates were collected from three locations: 1) the vineyards of the Department of Viticulture and Enology, University of California, Davis; 2) the National Clonal Germplasm Repository vineyard, Winters, CA; and 3) a private garden, Woodland, CA.
Multiple plants of susceptible $V$. vinifera cultivars 'Carignane' and 'Thompson Seedless' were maintained in a greenhouse to produce the leaves needed to culture $E$. necator. The third and fourth youngest leaves were collected from shoots and surface sterilized in $0.3 \%$ sodium hypochlorite solution for 2-3 min, rinsed four to five times in sterile distilled water, and dried between layers of sterile paper towels in a laminar flow hood. The petiole was cut to $\sim 0.5 \mathrm{~cm}$ in length, and placed onto a $0.8 \%$ agar medium in $100 \mathrm{~mm} \times$ $15 \mathrm{~mm}$ Petri dishes with the adaxial surface up and the cut petiole inserted into the medium. Conidia and mycelium from field collected infected leaves were used to inoculate surfacesterilized leaves using an eyelash attached to a small brush handle or to a Pasteur pipette. Three subsequent sub-transfers were made to new leaves using a single chain of conidia to ensure a pure colony was isolated. The resulting isolates were maintained on leaves at room temperature $\left(20\right.$ to $\left.25^{\circ} \mathrm{C}\right)$ and indirect light. Colonies were transferred to new leaves every 14-20 days.

Powdery mildew isolates with the branched conidiophore phenotype were observed from all three locations. Only one isolate was maintained for future comparative work. The new morphotype was compared with an isolate isolated from infected shoots of 'Carignane' plants growing in the UC Davis department vineyard, which is hereafter referred to as the $\mathrm{C}$-isolate. 

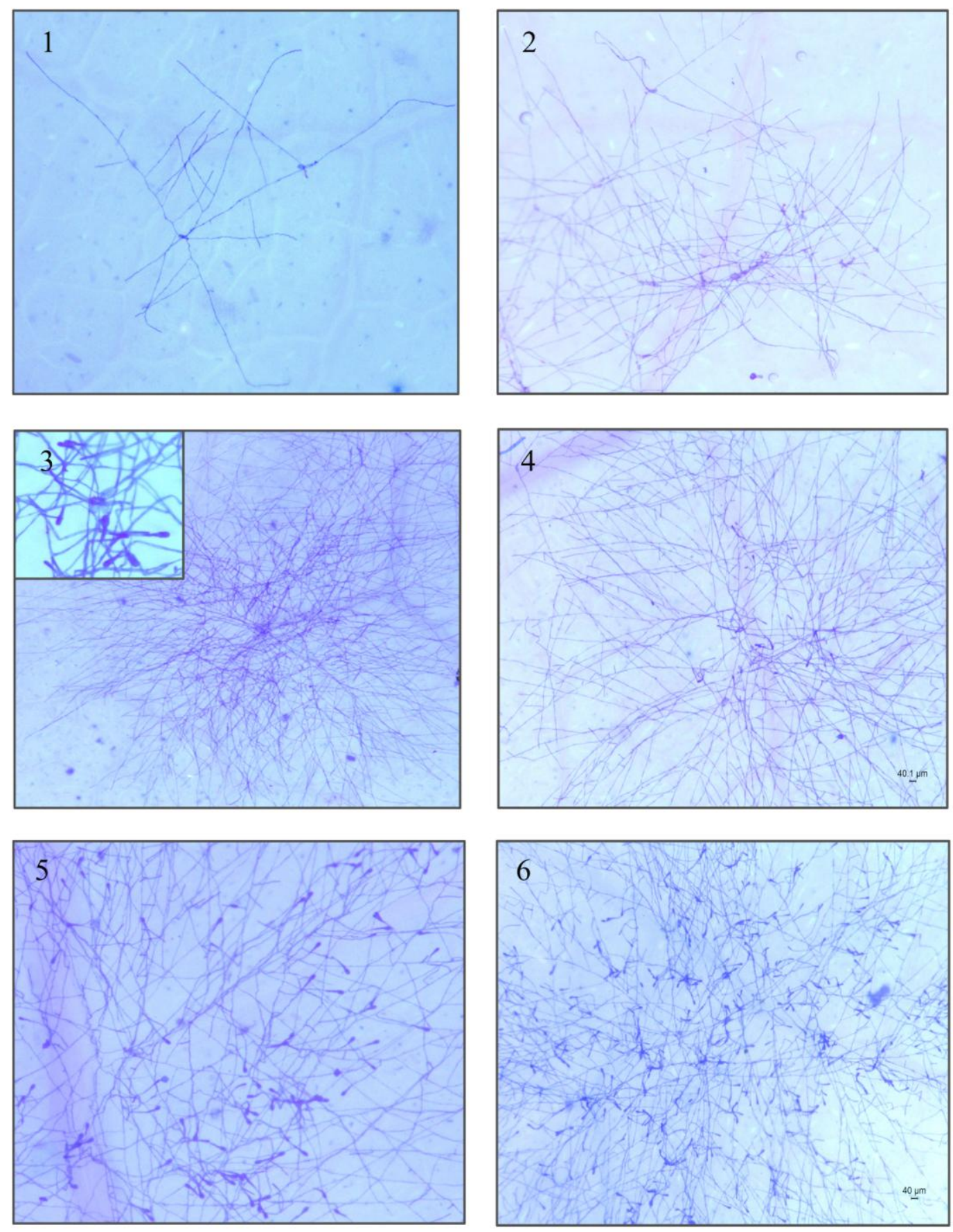

Fig. 2 - Development of both isolates. 1, 3, 5 Isolate with branched conidiophores at 72 hpi, 120 hpi and 7 dpi, respectively. 2, 4, 6 C-isolate at 72 hpi, 120 hpi and 7 dpi, respectively.

\section{Laboratory evaluations}

To compare the new morphotype to the C-isolate, 3-4 points on the adaxial surface of sterile leaves were inoculated with 1-2 conidial chains from a 10-day-old colony of Cisolate powdery mildew and a 18-day-old colony from the new morphotype. The inoculum was deposited gently on the recipient leaf surface without damaging the conidia. Inoculated leaves were placed in random order in transparent plastic boxes at room temperature and natural light conditions. Observations were made each morning from day 7 to day 18 on the live colonies with a Leica MC205C stereomicroscope and pictures were taken.

A second set of leaves was inoculated with both isolates and subjected to a Coomassie blue staining procedure adapted from Doster \& Schnathorst (1985). The $1.5 \mathrm{~cm}$ round leaf disks were cut using a brass pipe by keeping the point of inoculation at the center at 

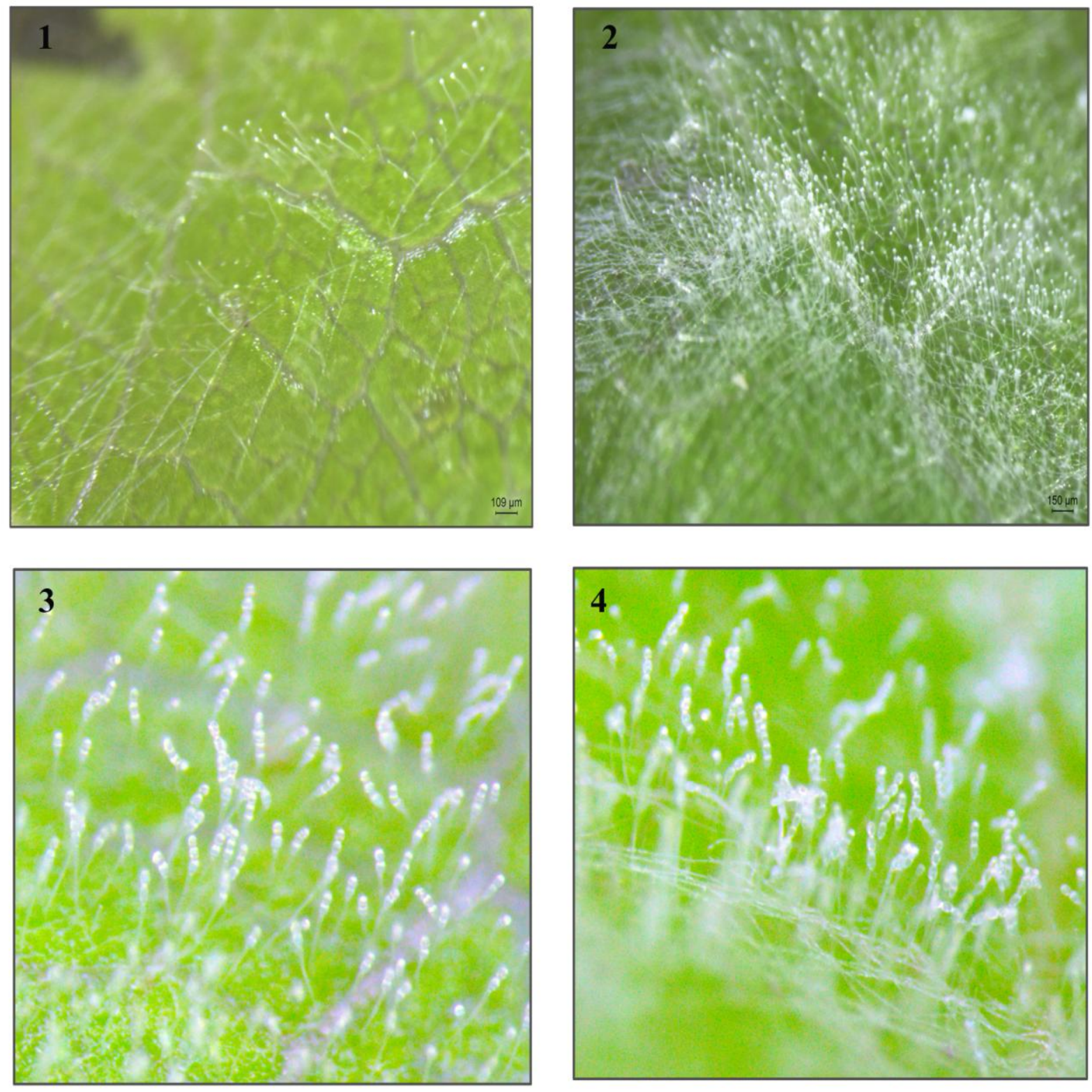

Fig. 3-1-2 Isolate with branched conidiophores at day 7. 3-4 C-isolate at day 7.

the following time intervals: 24,72 and 120 hours post inoculation (hpi), and 7, 11, 14 and 18 days post inoculation (dpi). Leaf disks were placed into a 6 -well plate filled with clearing solution (3:1 ethanol:acetic acid). This solution was changed two to three times until the tissue was completely bleached. Disks were then washed and placed in deionised water from one to four hours. Leaf disks were briefly dipped into Coomassie blue stain (Coomassie Brilliant Blue R-250, 0.12 g/liter, (Sigma-Aldrich, St. Louis, MO) in $50 \%$ (vol/vol) methanol, and $10 \%$ (vol/vol) glacial acetic acid) and rinsed with water followed by $50 \%$ glycerol, and then mounted for viewing on a microscope slide. The number of conidiophores was counted from mounted disks at 120 hours and 7 days after inoculation for both isolates.

\section{Results and Discussion}

There was no difference in the time to conidia germination. Conidia from both isolates developed primary germ tube, primary appressorium, primary hyphae, and elongated secondary hyphae from the appressorium (Fig. 1).

Seventy-two hours after inoculation, the new morphotype and the C-isolate had developed an extensive hyphal mat. Both isolates started producing conidiophores at 120 hpi (Fig. 2). For both isolates, the number of conidiophores increased normally between 120 

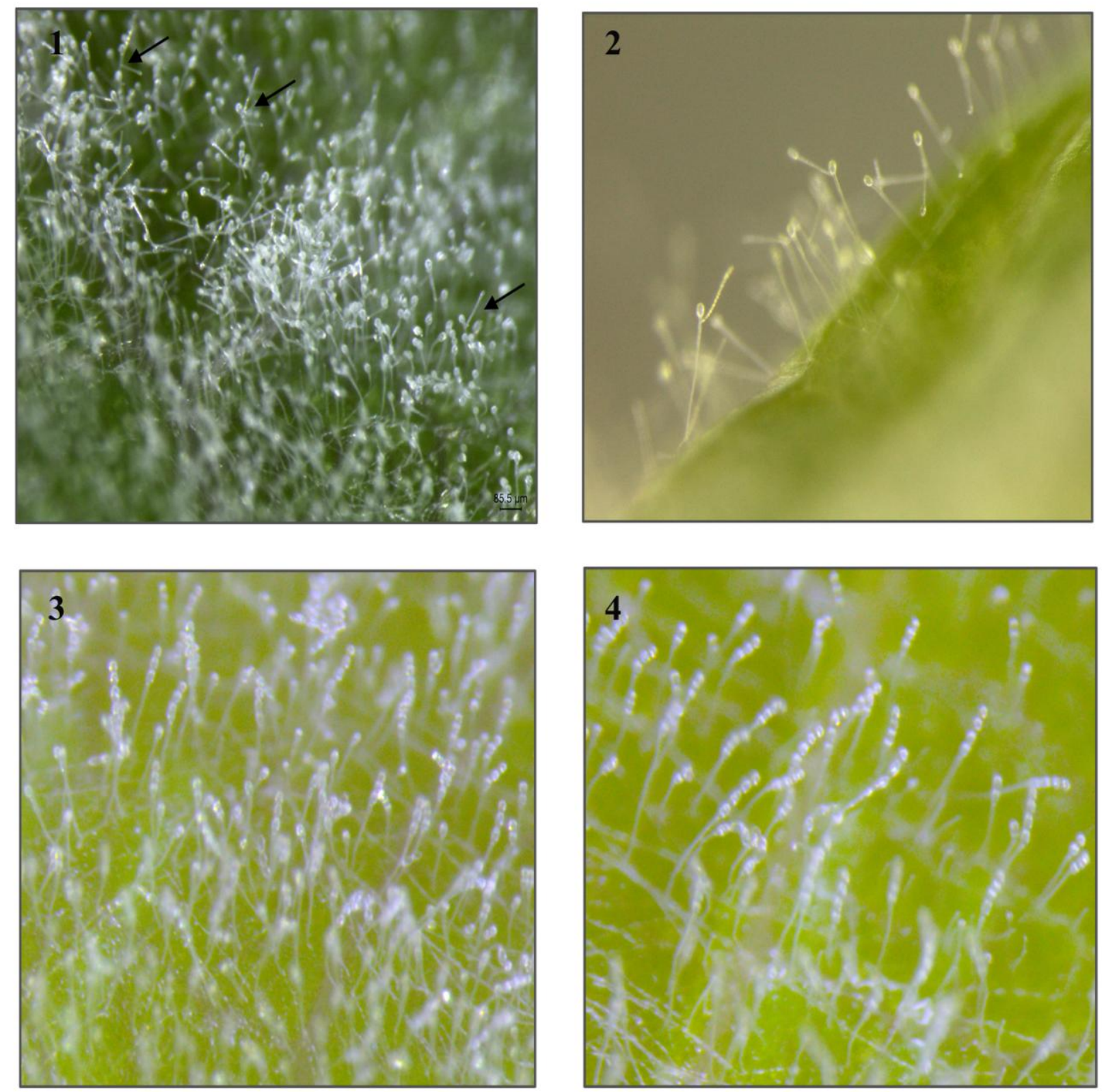

Fig. 4 - Comparison of both isolates at 10 dpi. 1-2 Isolate with branched conidiophores. Black arrow indicates branched conidiophores in the center of the colony. 3-4 C-isolate at day 10 .

hpi and $7 \mathrm{dpi}$, and differences in their number at these times were not significant.

Distinct differences in the appearance of the colonies of both isolates became evident on day 7. The C-isolate had long chains of conidia that were 2-3 conidia long (Fig. 3), while the new morphotype had only developed a foot cell with single conidia on top of it. This unusual arrested development in the new morphotype was observed in over 16 colonies from four different leaves.
From day 8 to 9 , more conidia were added to the $\mathrm{C}$-isolate conidiophores, which then had at least 3 conidia, while colonies of the new morphotype still had conidiophores with one conidium. At this point some of the new morphotype conidiophores were beginning to "branch" under the first conidia. By day 10, colonies of the new morphotype still had conidiophores with a single conidium, and many conidiophores had developed branches (Fig. 4). 

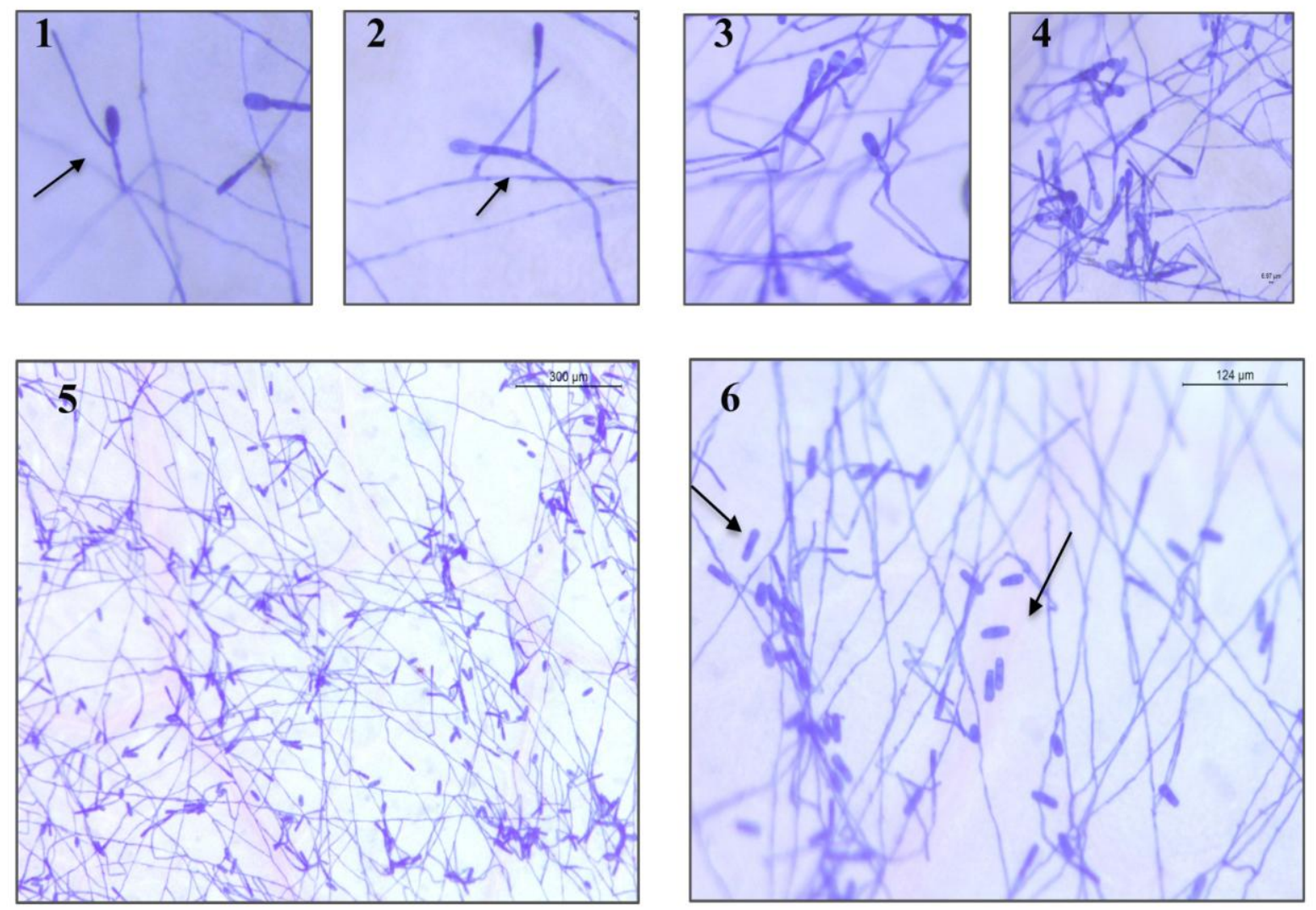

Fig. 5 - Slides prepared from leaf disks 11 dpi. 1-4 Close up of isolate with branched conidiophores showing examples of branches with one conidium at the tip. There was no evidence of free conidia that were dislodged during slide preparation. 5-6 Images of the C-isolate showing free conidia. Black arrows indicate attached and unattached conidia.

On $11^{\text {th }}$ day, the C-isolate colonies consisted primarily of $4-5$ conidia chains, but the new morphotype conidiophores still had a single conidium and most were branched. The conidiophores with branches were mostly in the middle of the colony. Observations of Coomassie blue stained slides found that $\mathrm{C}$ isolate conidiophores had lost most of their conidia during the disruptive slide preparation process and the majority of the conidiophore foot cells were devoid of conidia on the tip. Most of the conidia were loose and not attached to anything (Fig. 5), indicating that the $\mathrm{C}$-isolate conidia matured earlier and were more easily dislodged from other conidia in the chain. This observation also indicates that the conidia mature singly, i.e. they are not catenescent, and that conidia only adhere in "false chains". Meanwhile at this time colonies of the new morphotype were still at the stage where conidiophores consisted of only one conidium, they continued to make branches at the base of that conidium, and new branches were forming a conidium on the tip of the new branch. Free conidia were not observed on the new morphotype (Fig. 5). This observation indicates that the colonies of the new morphotype spend more energy and time making branches and less in producing mature conidia that can be dislodged from the branched conidiophores. This behavior puts this morphotype at a competitive disadvantage to normal isolates, which have long chains of easily detached conidia that readily start new colonies. Although the total number of conidia was not counted, isolates with branched conidiophores appeared to make fewer conidia compared to the C-isolate (Fig. 6).

On $16^{\text {th }}$ to $18^{\text {th }}$ day, C-isolate colonies were homogeneous and had conidiophores with 6-10 conidia in long straight chains (Fig. $7)$. On the $16^{\text {th }}$ day, the branched conidiophores of the new morphotype had elongated with 2-3 conidia each (Fig. 7). By the $18^{\text {th }}$ day (last day of observation) the new morphotype colonies were heteromorphic with the central part of the 

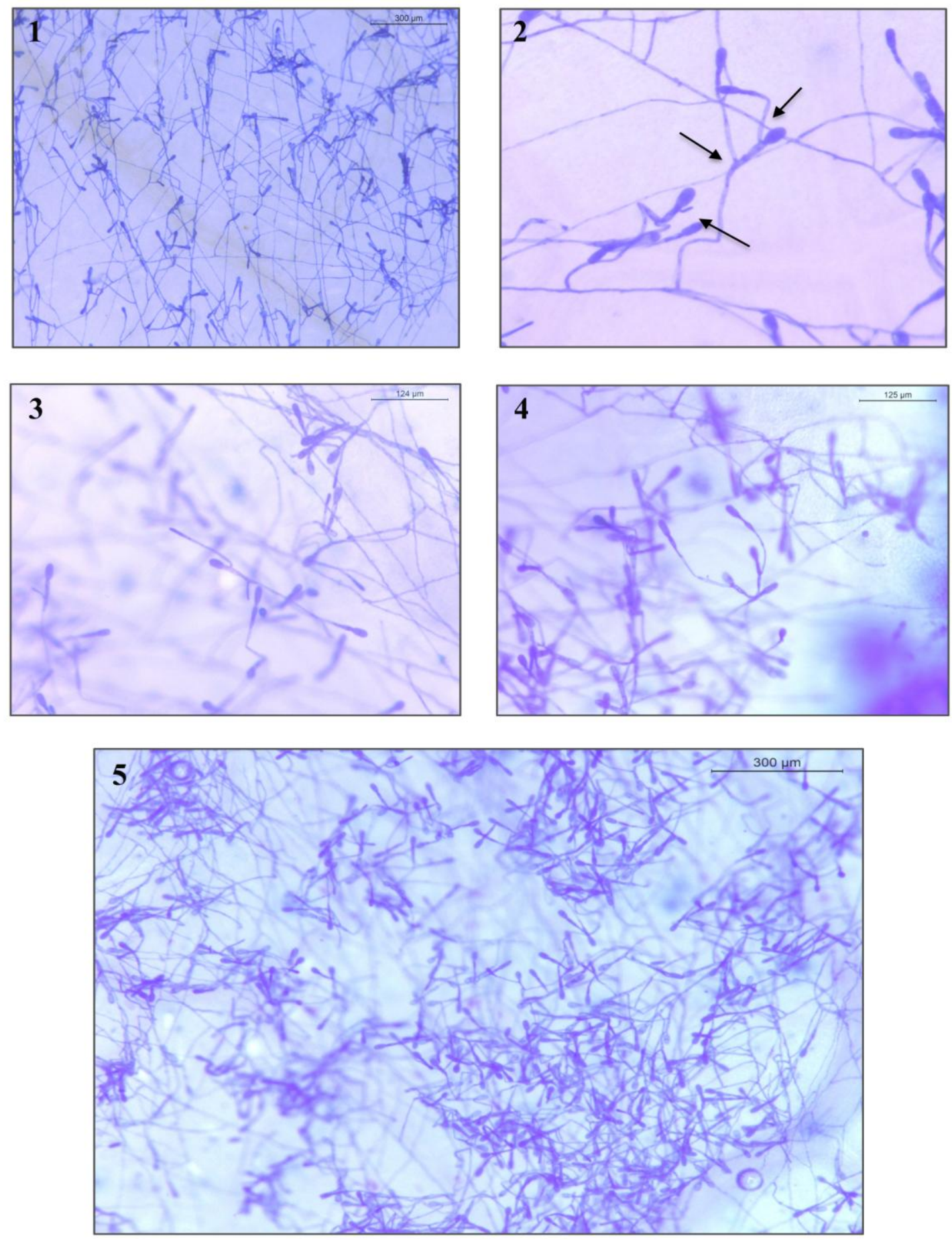

Fig. 6 - Slides prepared from the isolate with branched conidiophores. 1-2 Slides from 14 dpi. Black arrows indicate branches of conidiophores. 3-5 The same isolate 18 dpi.

colony having conidiophores with 1-3 conidia on top, and 3-4 conidia chains on the branches.

Non-branching conidiophores with 2-4 conidia were observed on the edges of the colonies. Conidia on the new morphotype were hard to separate from the chain until day 18, implying that these conidia take longer to mature and their adhesion to sister conidia in the chain is stronger.

Chains of conidia were taken from the main trunk and side branches of the conidiophores as well as from normal straight chains to initiate new colonies; all three types germinated and produced colonies that were a mix of both branched and non-branching conidiophores, as colonies aged. 

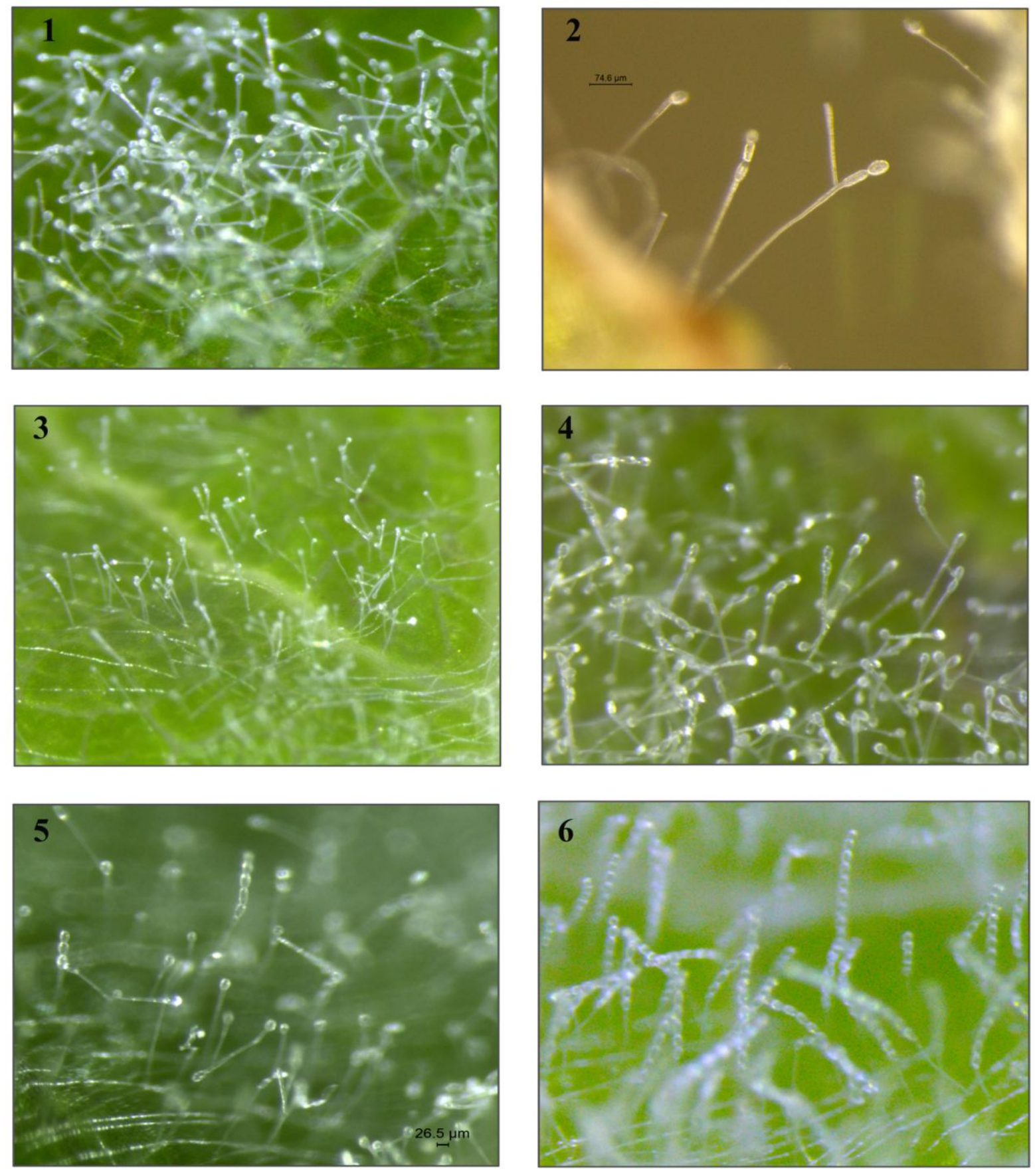

Fig. 7 - Pictures of live colonies of the two isolates. 1, 2. At 13 dpi the isolate with the branched conidiophores has many conidiophores and has started producing 2 conidia long chains. 3, 4. At 16 dpi the branched conidiophore isolate has 2 or 3 conidia long chains. 5. Isolate with the branched conidiophores at 18 dpi. 6. C-isolate with 10-16 conidia long chains at 18 dpi.

\section{Conclusion}

This report is the first to detail the appearance of a new morphotype of grapevine powdery mildew that makes branched conidiophores. The new morphotype produces branches that have short chains of conidia, which take a longer time to mature as compared to the normal unbranched powdery mildew isolate.

\section{Acknowledgements}

The authors gratefully acknowledge the funding support of the American Vineyard Foundation and the Louis P. Martini Endowed Chair research funds. 


\section{Reference}

Braun U, Cook RTA. 2012 - Taxonomic Manual of the Erysiphales (Powdery Mildews). CBS Biodiversity Series No. 11. CBS, Utrecht, Netherlands.

Cook RTA, Braun U. 2009 - Conidial germination patterns in powdery mildews. Mycological Research 113, 616-636.

Doster MA, Schnathorst WC. 1985 - Effects of leaf maturity and cultivar resistance on development of the powdery mildew fungus on grapevines. Phytopathology 75, 318-321.

Gadoury DM, Cadle-Davidson L, Wilcox WF, Dry IB, Seem RC, Milgroom MG. 2012 - Grapevine powdery mildew
(Erysiphe necator): a fascinating system for the study of the biology, ecology, and epidemiology of an obligate biotroph. Molecular Plant Pathology 13, 1-16.

Kiss L, Jankovics T, Kovacs GM, Daughtrey ML. 2008 - Oidium longipes, a new powdery mildew fungus on petunia in the USA: a potential threat to ornamental and vegetable solanaceous crops. Plant Disease 92, 818-825.

Takikawa Y, Xu L, Kakutani K, Nonomura T, Sameshima T, Matsuda Y, Toyoda H. 2011 - Conidia of the tomato powdery mildew Oidium neolycopersici initiate germ tubes at a predetermined site. Mycoscience 52, 198-203 